\title{
Differences in TNF- $\alpha$ and TNF-R1 expression in damaged neurons and activated astrocytes of the hippocampal CA1 region between young and adult gerbils following transient forebrain ischemia
}

\author{
CHOONG HYUN LEE ${ }^{1 *}$, JI HYEON AHN ${ }^{2,3 *}$, BAI HUI CHEN ${ }^{4}$, DAE WON KIM ${ }^{5}$, HYEJIN SIM ${ }^{3}$, \\ TAE-KYEONG LEE ${ }^{6}$, JOON HA PARK ${ }^{7}$, MOO-HO WON ${ }^{3}$ and SOO YOUNG CHOI ${ }^{6}$
}

\author{
${ }^{1}$ Department of Pharmacy, College of Pharmacy, Dankook University, Cheonan, Chungnam 31116; \\ ${ }^{2}$ Department of Physical Therapy, College of Health Science, Youngsan University, Yangsan, Gyeongnam 50510; \\ ${ }^{3}$ Department of Neurobiology, School of Medicine, Kangwon National University, Chuncheon, Gangwon 24341, \\ Republic of Korea; ${ }^{4}$ Department of Histology and Embryology, Institute of Neuroscience, Wenzhou Medical University, \\ Wenzhou, Zhejiang 325035, P.R. China; ${ }^{5}$ Department of Biochemistry and Molecular Biology, \\ and Research Institute of Oral Sciences, College of Dentistry, Gangnung-Wonju National University, Gangneung, \\ Gangwon 25457; ${ }^{6}$ Department of Biomedical Science, Research Institute of Bioscience and Biotechnology, \\ Hallym University, Chuncheon, Gangwon 24252; ${ }^{7}$ Department of Anatomy, College of Korean Medicine, \\ Dongguk University, Gyeongju, Gyeongbuk 38066, Republic of Korea
}

Received April 13, 2021; Accepted June 2, 2021

DOI: $10.3892 / \mathrm{mmr} .2021 .12264$

\begin{abstract}
Tumor necrosis factor (TNF)- $\alpha$ and TNF receptor 1 (TNF-R1) play diverse roles in modulating the neuronal damage induced by cerebral ischemia. The present study compared the time-dependent changes of TNF- $\alpha$ and TNF-R1 protein expression levels in the hippocampal subfield cornu ammonis 1 (CA1) between adult and young gerbils following transient forebrain ischemia (tFI), via western blot and immunohistochemistry analyses. In adult gerbils, delayed neuronal death of pyramidal neurons, the principal neurons in CA1, was recorded 4 days after tFI; however, in young gerbils, delayed neuronal death was recorded 7 days after tFI. TNF- $\alpha$ protein expression levels gradually increased in both groups following tFI; however, TNF- $\alpha$ expression was
\end{abstract}

Correspondence to: Professor Moo-Ho Won, Department of Neurobiology, School of Medicine, Kangwon National University, 1 Kangwondaehak Gil, Chuncheon, Gangwon 24341, Republic of Korea

E-mail: mhwon@kangwon.ac.kr

Professor Soo Young Choi, Department of Biomedical Science, Research Institute of Bioscience and Biotechnology, Hallym University, 1 Hallymdaehak Gil, Chuncheon, Gangwon 24252, Republic of Korea

E-mail: sychoi@hallym.ac.kr

*Contributed equally

Key words: tumor necrosis factor- $\alpha$, tumor necrosis factor-receptor 1 , transient forebrain ischemia, delayed neuronal death, pyramidal neuron, astrocyte, age higher in young gerbils compared with adult gerbils. TNF-R1 protein expression levels markedly increased in both groups 1 day after tFI. Subsequently, TNF-R1 expression gradually decreased in young gerbils, whereas TNF-R1 expression levels were irregularly altered in adult gerbils following tFI. Notably, TNF- $\alpha$ immunoreactivity significantly increased in pyramidal neurons in both groups 1 day after $\mathrm{tFI}$; however, the patterns altered between both groups. In adult gerbils, TNF- $\alpha$ immunoreactivity was rarely exhibited in pyramidal neurons 4 days after tFI due to neuronal death, suggesting that TNF- $\alpha$ immunoreactivity was newly expressed in astrocytes. In young gerbils, TNF- $\alpha$ immunoreactivity increased in pyramidal neurons 4 days after tFI, and TNF- $\alpha$ immunoreactivity was newly expressed in astrocytes. In addition, TNF-R1 immunoreactivity was exhibited in pyramidal cells of both sham groups, and significantly increased 1 day after tFI; however, the patterns altered between both groups. In adult gerbils, TNF-R1 immunoreactivity was rarely exhibited 4 days after tFI, and astrocytes newly expressed TNF-R1 immunoreactivity. In young gerbils, TNF-R1 immunoreactivity increased in pyramidal neurons 4 days after tFI; however, TNF-R1 immunoreactivity was not reported in pyramidal neurons and astrocytes thereafter. Taken together, the results of the present study suggest that different expression levels of TNF- $\alpha$ and TNF-R1 in ischemic CA1 between adult and young gerbils may be due to age-dependent differences of tFI-induced neuronal death.

\section{Introduction}

Transient forebrain ischemia (tFI) causes the pathophysiological alterations and a selective neuronal death in the brain. Especially, the pyramidal neurons in the hippocampal cornu ammonis 1 (CA1) region are well known to be the 
most vulnerable to tFI (1). tFI-induced neuronal death in the pyramidal neurons of hippocampal CA1 region is called as the delayed neuronal death (DND), because it occurs a few days following tFI (1). To explain the tFI-induced DND, many underlying mechanisms has been suggested, including neuroinflammatory processes. Following cerebral ischemia, neuroinflammatory responses and upregulation of inflammatory cytokines occurs generally in the brain, and the imbalance between pro-inflammatory cytokines, such as tumor necrosis factor (TNF)- $\alpha$, and anti-inflammatory cytokines could lead to the neuronal damage after ischemic injury (2-5). In addition, the cerebral ischemia-induced neuronal damage has been also known to be affected by various factors, including ischemic duration, the age and sex of experimental animals (6-9).

TNF- $\alpha$ plays a diverse biological role in regulating inflammatory responses, and its biological actions is mediated through two plasma membrane receptors, TNF receptor 1 (TNF-R1) and TNF-R2, which are present in all cell types, including neurons and glial cells, in the CNS (10-12). It has been well known that toxic effects of TNF- $\alpha$ begin with and require binding to TNF-R1, and that TNF-R1 is responsible for mediating the detrimental effects of TNF- $\alpha$, such as apoptotic cell death and cytokine production $(13,14)$. However, another study also reported that the protective role of TNF- $\alpha$ against excitotoxic hippocampal neurodegeneration was closely associated with TNF-R1 signaling (15). In addition, TNF- $\alpha$ has been thought to be one of important mediators in modulating neuronal death in in vitro and in vivo models of ischemic damage $(13,16,17)$.

In our previous studies, we reported the tFI-induced changes of TNF- $\alpha$ and/or TNF-R1 protein expression in the hippocampus of adult gerbils $(4,18,19)$. However, the relationship between TNF- $\alpha$ and neuronal damage in the hippocampus of young animals has not been fully elucidated yet. Therefore, in the present study, we compared the time-dependent changes of TNF- $\alpha$ and TNF-R1 protein expression in the hippocampal CA1 region of adult and young gerbils after $\mathrm{tFI}$.

\section{Materials and methods}

Experimental animals. Male gerbils of one month (body weight: $25-30 \mathrm{~g}$ ) and six months (body weight: $65-75 \mathrm{~g}$ ) of age were used as young and adult groups. The animals were provided by the Experimental Animal Center of Kangwon National University (Chuncheon, Kangwon, Republic of Korea). They were housed in a conventional state (room temperature, $23 \pm 0.5^{\circ} \mathrm{C}$; humidity, $55 \pm 5 \%$; $12: 12$ light/dark cycle) with freely accessible pellet feed and water. The protocol of this experiment was approved (approval no. KW-200113-1) by the Institutional Animal Care and Use Committee.

Experimental groups and induction of tFI. The gerbils used in this research were divided into four groups: i) adult sham group ( $\mathrm{n}=28)$; ii) tFI-operated adult gerbils (adult ischemia group) $(n=56)$; iii) young sham group $(n=28)$; and iv) young ischemia group $(n=56)$.

As previously described in our published papers $(4,19,20)$, the surgical procedure of $\mathrm{tFI}$ in the gerbils was performed as follows. Briefly, the gerbils were adequately anesthetized using mixture of $2.5 \%$ isoflurane (Baxtor) in $67 \%$ nitrous oxide and $33 \%$ oxygen (21). Under the anesthesia, both common carotid arteries, which locate at the neck and supply arterial blood to the brain, were isolated and ligated for $5 \mathrm{~min}$ with aneurysm clips. Body temperature was checked using a rectal temperature probe and controlled at normothermic condition $\left(37 \pm 0.5^{\circ} \mathrm{C}\right)$ using a thermometric blanket before and during tFI. After tFI, body temperature was controlled at normothermia until they awaked. The sham gerbils received the same tFI operation without the occlusion of the arteries.

Western blotting for TNF- $\alpha$ and TNF-Rl. The gerbils in the sham groups were sacrificed at 1 day $(n=7)$ and 4 days $(n=7$, data not shown) after tFI, the gerbils in the ischemia groups were sacrificed at 1 day $(n=7), 4$ days $(n=7), 7$ days $(n=7)$ and 15 days $(n=7)$ after tFI. The gerbils in all groups were deeply anesthetized with $70 \mathrm{mg} / \mathrm{kg}$ pentobarbital sodium, and the dose was increased to $200 \mathrm{mg} / \mathrm{kg}$ for euthanasia. The confirmation of death was evaluated with vital signs including heart beats, pupillary response, and respiratory pattern (lack of cardiac activity for $5 \mathrm{~min}$ through cardiac palpation, unresponsiveness to light with dilated pupils using light into the eyes of the animal, and lack of spontaneously breathing pattern with shallow and irregular breathing pattern). As previously described (22), hippocampal proteins (50 $\mu \mathrm{g}$ for each sample) were denatured, electrophoretically separated, and transferred to a nitrocellulose membranes. The membrane was blocked with TBS-T buffer containing non-fat dry milk (5\%) and incubated for $8 \mathrm{~h}$ at $4^{\circ} \mathrm{C}$ with primary antibodies-rabbit anti-TNF- $\alpha$ or rabbit anti-TNF-R1 (diluted 1:1,000; Abcam). The next day, the membrane was incubated with secondary antibody [peroxidase conjugated goat anti-rabbit IgG (Pierce; Thermo Fisher Scientific)] for $1 \mathrm{~h}$ at room temperature. Thereafter, the membranes was analyzed using chemiluminescence system according to the manufacturer (Amersham; GE Healthcare.).

The western blot bands of TNF- $\alpha$ and TNF-R1 were scanned by ChemiDoc Imaging System (Bio-Rad Laboratories, Inc. The protein signal was quantified by scanning densitometry using Scion Image software of Scion Corp. Data were normalized to appropriate references ( $\beta$-actin for total protein).

Preparation of histological sections. The gerbils in the sham groups were sacrificed at 1 day $(n=7)$ and 4 days $(n=7$, data not shown) after tFI, and the gerbils in the ischemia groups were sacrificed at 1 day $(n=7), 4$ days $(n=7), 7$ days $(n=7)$ and 15 days $(n=7)$ after tFI. In our current study, to reduce the numbers of the gerbils, the brain sections of the young and adult sham groups were obtained at 1 and 4 days after sham operation. The brain sections containing the hippocampi were prepared as previously described in our studies $(4,19,20)$. In brief, the gerbils in all groups were deeply anesthetized with 70 90 mg/kg pentobarbital sodium (21) at 1, 4, 7 and 15 days after tFI. Under anesthesia, the gerbils were perfused transcardially with saline, and then with $4 \%$ paraformaldehyde solution. The brains were obtained and more fixed in the same fixative for $6 \mathrm{~h}$ at room temperature. For cutting the brains, they were infiltrated with $30 \%$ sucrose solution to avoid tissue damage from freeze. Finally, the brain tissues were coronally sectioned into $30-\mu \mathrm{m}$ thickness in a cryostat.

Fluoro-Jade B (FJB) histofluorescence. To examine neuronal damage/death (loss) in CA1 after tFI, FJB histofluorescence 
was done as described previously (23). In short, the tissues (sections) were incubated in $0.06 \%$ potassium permanganate and stained with $0.0004 \%$ FJB (Histochem) for 10 min using a rotator. Thereafter, the sections were rinsed and incubated in $0.0004 \%$ FJB (Histochem) for 20 min After washing, the sections were placed on a slide warmer for the reaction of F-J B. Thereafter, the sections were fully dried, cleared by immersion in xylene and coverslipped with dibutylphthalate polystyrene xylene (DPX; Sigma-Aldrich; Merck KGaA).

To count the FJB positive cells (neurons) in CA1, five sections/gerbil were chosen and analyzed using previously described method $(4,19,20)$. The image of FJB positive cells was taken using fluorescence microscope from Carl Zeiss with blue excitation fluorescence filter between 450-490 nm. The FJB positive cells were captured using image capture software (cellSens Standard; Olympus). The captured FJB positive cells were totally counted in $250 \mu \mathrm{m}^{2}$ at the middle in CA1. The mean number was calculated using NIH Image 1.59 software (NIH; National Institutes of Health).

Immunohistochemistry for neuronal nuclear antigen (NeuN), $T N F-\alpha$ and TNF-R1. According to published methods $(4,19,20)$ with modification, immunohistochemical staining using a single antibody (NeuN, TNF- $\alpha$ and TNF-R1) was performed by streptavidin-biotin-peroxidase method. In brief, the sections were immersed with $0.3 \%$ hydrogen peroxide to block endogenous peroxidase activity ( $20 \mathrm{~min}$ at room temperature), and $5 \%$ normal horse serum was treated to block unspecific proteins in the tissues (30 min at room temperature). And then, the tissues were incubated with each antibody-mouse anti-NeuN $(1: 1,100$, Chemicon), rabbit anti-TNF- $\alpha$ (1:1,100, Abcam) or rabbit anti-TNF-R1 (1:1,000, Abcam)-for $10 \mathrm{~h}$ at $4^{\circ} \mathrm{C}$. The tissues were rinsed and incubated with biotinylated horse anti-mouse or anti-rabbit IgG (Vector Laboratories, Inc.) for $2 \mathrm{~h}$ at room temperature, and continuously reacted with Elite avidin-biotin enzyme complex (ABC; Vector Laboratories, Inc.) for $1 \mathrm{~h}$ at room temperature. The visualization of each immunoreaction was achieved with solution of 3,3'-diaminobenzidine (Vector Laboratories, Inc.).

To count NeuN-immunoreactive cells, and to analyze TNF- $\alpha$ - and TNF-R1-immunoreactive structure, we used published methods $(4,19,20)$ In short, five sections/gerbil with $120-\mu \mathrm{m}$ interval were chosen. The digital images of NeuN-immunoreactive cells were captured in $250 \mu \mathrm{m}^{2}$ at the middle of CA1 using light microscope (BX53) from Olympus. For TNF- $\alpha$ - and TNF-R1-immunoreactive structure, their digital images were also captured in all layers using BX53 microscope. TNF- $\alpha$ - and TNF-R1-immunoreactivity was evaluated by relative optical density (ROD) according to published methods $(19,20)$ with some modification. Namely, the color image was converted to 8 -bit greyscale images with a rage of 0-255 (from black to white). The background level and the variance of the staining intensity in CA1 was calculated on the 0-255 greyscale. The percentile in the corresponding area was analyzed using image analysis software from Image $\mathrm{J}(\mathrm{NIH})$.

Double immunofluorescence. To confirm the type of TNF- $\alpha$ - and TNF-R1-immunoreactive cells, double immunofluorescence was performed using rabbit anti-TNF- $\alpha$ (diluted 1:400; Abcam)/mouse anti-GFAP (diluted 1:1,000, Chemicon) or rabbit anti-TNF-R1 (diluted 1:400, Abcam)/mouse anti-GFAP (diluted 1:1,000, Chemicon) for astrocytes. The sections obtained at 7 days after $\mathrm{tFI}$ were incubated in the mixture of antisera for $8 \mathrm{~h}$ at $4^{\circ} \mathrm{C}$. They were briefly washed and reacted with the mixture of both Cy3- or FITC-conjugated donkey anti-rabbit or anti-mouse IgG (diluted 1:200; Jackson ImmunoResearch) for $2 \mathrm{~h}$ at room temperature. The double immunoreaction was examined with confocal MS from LSM510 META NLO (Carl Zeiss).

Statistical analysis. The data presented in this study represent the means \pm SEM. The data were statistically analyzed using SPSS 18.0 (SPSS, Inc.). The two-way analysis of variance (ANOVA) with a post hoc Bonferroni's multiple comparison test was done to determine differences among groups. $\mathrm{P}<0.05$ was used for statistical significance.

\section{Results}

Difference in tFI-induced DND between the adult and young. We examined the difference of tFI-induced DND in CA1 of gerbil hippocampus between adult and young gerbils using neuronal nuclear antigen (NeuN, a marker for neuron) immunohistochemistry and Fluoro-Jade B (FJB, a marker for degenerating or dead neuron) histofluorescence (Fig. 1).

In adult and young sham groups, NeuN immunoreactivity was easily identified in pyramidal neurons $\left(88.0 \pm 3.8 / 250 \mu \mathrm{m}^{2}\right)$ located in the stratum pyramidale (SP) (Fig. 1A and 1B). In addition, FJB-positive cells were not detected in SP (Fig. 1A and B). This finding means that no damaged cells were present in the sham groups.

In adult ischemia group, a significant loss of NeuN-immunoreactive CA1 pyramidal neurons $\left(11.3 \pm 1.9 / 250 \mu \mathrm{m}^{2}\right)$ and many FJB-positive neurons $\left(78.1 \pm 1.7 / 250 \mu \mathrm{m}^{2}\right)$ were observed in SP at 4 days after tFI. In this group, the number of $\mathrm{NeuN}$-immunoreactive neurons was approximately $13 \%$ of that of the adult sham group (Fig. 1C and Cc). Thereafter, the numbers of NeuN-immunoreactive neurons and FJB-positive cells at 7 and 15 days after tFI were similar to those at 4 days after tFI (Fig. 1E, Ee, G, Gg, I and J).

In the young ischemia group, the number of NeuN-immunoreactive pyramidal neurons at 4 days after tFI was similar to that in young sham group, although the NeuN immunoreactivity was decreased (Fig. 1D, Dd and I). However, at 7 and 15 days after $\mathrm{tFI}$, the number of NeuN-immunoreactive pyramidal neurons was distinctly decreased (approximately 48.5 and $47.9 \%$, respectively), compared with that in the young sham group (Fig. 1F, H and I). In addition, we found that many FJB-positive pyramidal neurons were observed at 7 and 15 days after $\mathrm{tFI}$, showing that the mean percentage of the neurons was approximately 57 and $55 \%$, respectively, of that in the adult ischemia group, respectively (Fig. 1f, h and J).

Difference in tFI-induced changes of TNF- $\alpha$ and TNF-RI levels between the adult and young. To compare the changes of TNF- $\alpha$ and TNF-R1 protein levels in ischemic CA1 between adult and young gerbils, western blot was conducted in CA1 after tFI (Fig. 2).

Protein levels of TNF- $\alpha$ in the adult and young CA1 were significantly changed with time after tFI (Fig. 2). In both 

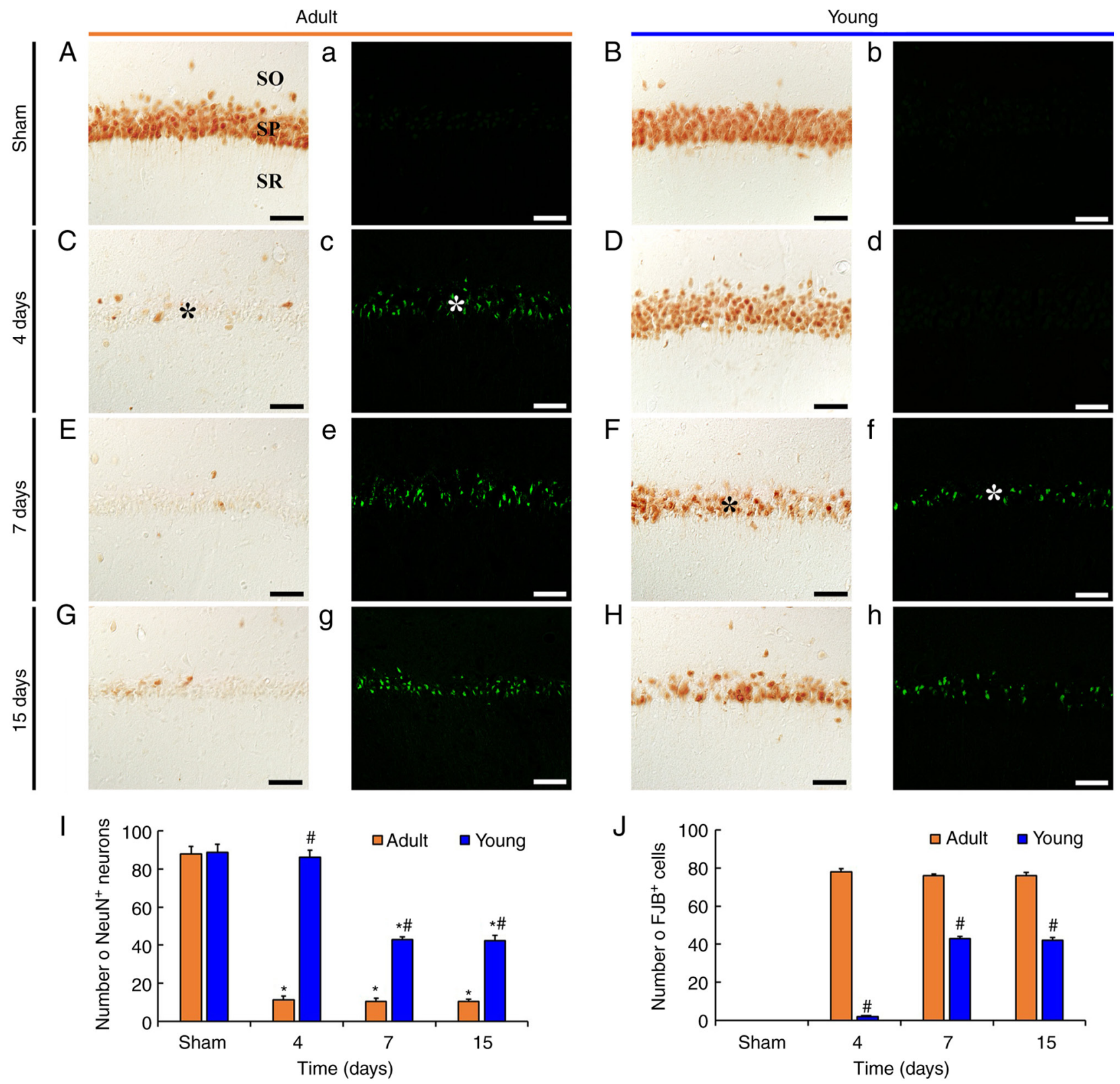

Figure 1. tFI-induced delayed neuronal death. (A-H) NeuN immunohistochemistry and (a-h) FJB histofluorescence staining in the CA1 in the (A, a, B and b) sham and (C-H and c-h) ischemia groups of adult (left two columns) and young (right two columns) gerbils. In the adult ischemia group, a few NeuN-immunoreactive pyramidal neurons and several FJB-positive pyramidal neurons were observed in the SP (asterisks) 4 days after tFI. In the young ischemia group, the number of NeuN-immunoreactive neurons significantly decreased and FJB-positive neurons increased 7 days after tFI. Scale bar, $50 \mu \mathrm{m}$. (I and J) Mean numbers of NeuN-immunoreactive and FJB-positive neurons in $250 \mu \mathrm{m}^{2}$ at the center of the CA1, respectively. Data are presented as the mean \pm SEM. ${ }^{*} \mathrm{P}<0.05$ vs. sham group; ${ }^{\# P}<0.05$ vs. corresponding adult group. NueN, neuronal nuclear antigen; FJB, Fluoro-Jade B; CA1, cornu ammonis 1 ; tFI, transient forebrain ischemia; SP, stratum pyramidale; SO, stratum oriens; SR, stratum radiatum.

groups, TNF- $\alpha$ protein levels were gradually increased from 1 day after tFI, however, TNF- $\alpha$ protein levels were significantly higher in the young group than that in the adult group, showing that relative density in the young was 1.1-fold at 1 day, 1.5 -fold at 4 days, 1.2 -fold at 7 days and 1.6-fold at 15 days after $\mathrm{tFI}$ compared with that in the corresponding adult groups (Fig. 2).

The change pattern of TNF-R1 protein levels in the adult and young was not similar to the changes of TNF- $\alpha$ protein levels, but the changes were significant (Fig. 2). At 1 day after tFI, TNF-R1 protein levels were dramatically increased in both groups (2.1-fold in the adult and 2.1-fold in the young) compared with those in each sham group (Fig. 2). Thereafter, TNF-R1 protein levels in both groups were irregularly altered, showing that relative density in the young groups was 1.7-fold at 4 days, 0.4-fold at 7 days, and 0.5 -fold at 15 days, respectively, after tFI compared with that in the corresponding adult groups (Fig. 2).

Difference in $\mathrm{tFI}$-induced change of TNF- $\alpha$ immunoreactivity between the adult and young. To investigate the change of TNF- $\alpha$ immunoreactivity in CA1, immunohistochemistry for TNF- $\alpha$ was conducted in adult and young gerbils after tFI (Fig. 3). 


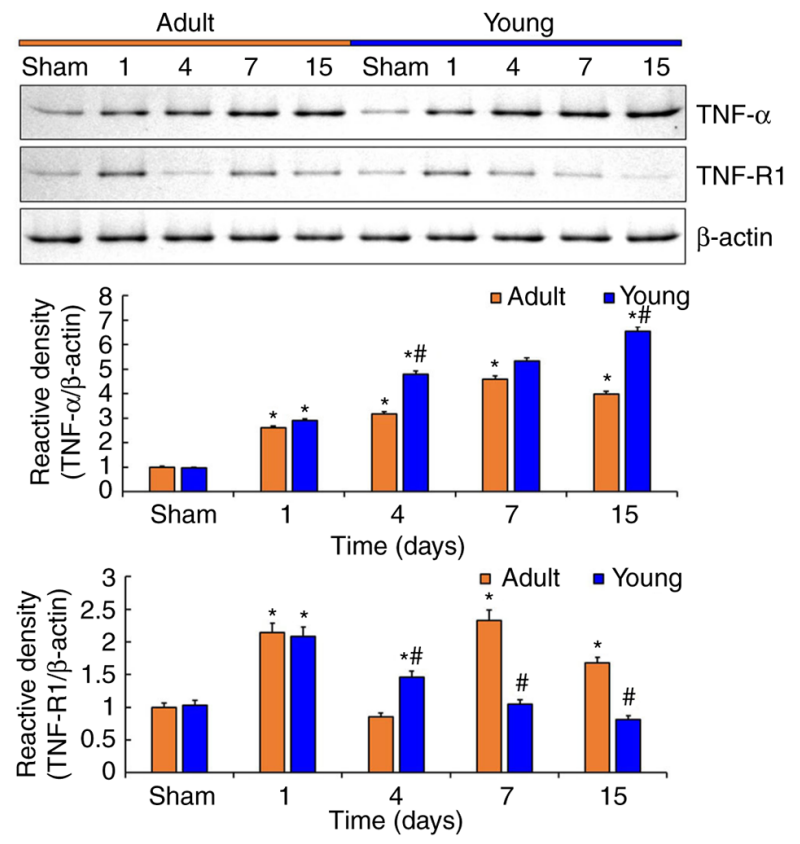

Figure 2. Representative blots and quantitative analyses of TNF- $\alpha$ and TNF-R1 protein expression levels in the CA1 in the sham and ischemia groups of adult and young gerbils. Data are presented as the mean \pm SEM $(\mathrm{n}=7) .{ }^{*} \mathrm{P}<0.05$ vs. sham group; ${ }^{*} \mathrm{P}<0.05$ vs. corresponding adult group. TNF, tumor necrosis factor; R1, receptor 1; CA1, cornu ammonis 1 .

Weak TNF- $\alpha$ immunoreactivity was mainly found in CA1 pyramidal neurons in the adult sham group (Fig. 3A and K). In the adult ischemia group, a significant increase of TNF- $\alpha$ immunoreactivity (172.5\% of the adult sham group) was found in the pyramidal neurons at 1 day after $\mathrm{tFI}$, compared with that in the adult sham group (Fig. 3C and K). At 4 days after tFI, TNF- $\alpha$ immunoreactivity was hardly observed in the pyramidal neurons; however, at this time, TNF- $\alpha$-immunoreactivity began to be expressed in non-pyramidal cells located in the stratum oriens (SO) and stratum radiatum (SR) (Fig. 3E and K). Thereafter, TNF- $\alpha$-immunoreactivity was mainly observed in the non-pyramidal cells, and the relative optical density (ROD) of the TNF- $\alpha$-immunoreactivity in CA1 was markedly increased (339.1\% in the adult and $357.4 \%$ in the young) at 7 and 15 days after tFI compared to that in the adult sham group (Fig. 3G, I and K).

In the young sham group, no significant difference in TNF- $\alpha$ immunoreactivity in the pyramidal neurons was found when compared with that in the adult sham group (Fig. 3B and $\mathrm{K}$ ). In the young ischemia group, $\mathrm{TNF}-\alpha$ immunoreactivity in the pyramidal was significantly increased at 1 and 4 days after $\mathrm{tFI}$, and the ROD in CA1 was increased (161.2\% in the adult and $156.7 \%$ in the young) compared to that in the corresponding adult ischemia group (Fig. 3D, F and K). At 7 and 15 days after tFI, TNF- $\alpha$ immunoreactivity in the pyramidal neurons was still observed, and non-pyramidal cells newly expressed TNF- $\alpha$ immunoreactivity, showing that the ROD in CA1 was increased (117.4\% in the adult and $125.8 \%$ in the young) compared to that in the corresponding adult ischemia group (Fig. 3H, J and K).

As described above, TNF- $\alpha$ immunoreactivity was shown in non-pyramidal cells at late time after tFI. To identify the cell types of the non-pyramidal cells containing TNF- $\alpha$

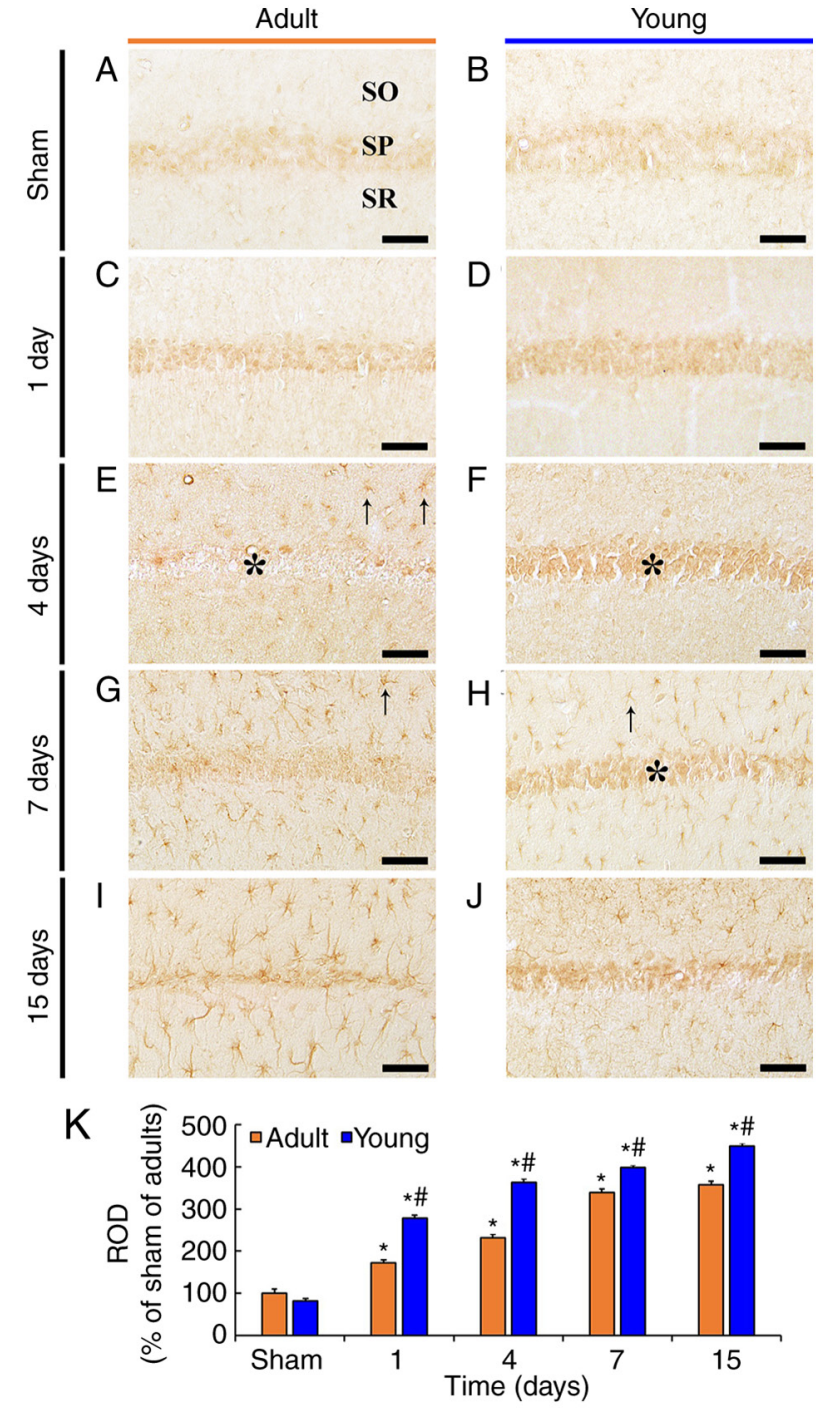

Figure 3. TNF- $\alpha$ immunohistochemistry. (A-J) TNF- $\alpha$ immunohistochemistry in the CA1 in the (A and B) sham and (C-J) ischemia groups of adult (left column) and young (right column) gerbils. In the adult ischemia group, TNF- $\alpha$ immunoreactivity was rarely exhibited in the SP (asterisks) and observed in non-pyramidal cells (arrows) located in the SO and SR 4 days after tFI. In the young ischemia group, TNF- $\alpha$ immunoreactivity in the SP was observed 15 days after tFI, whereas TNF- $\alpha$ immunoreactivity was observed in non-pyramidal cells 7 days after tFI. Scale bar, $50 \mu \mathrm{m}$. (K) ROD as $\%$ of TNF- $\alpha$ immunoreactivity in CA1 $(n=7)$. ${ }^{*} \mathrm{P}<0.05$ vs. sham group; ${ }^{~} \mathrm{P}<0.05$ vs. corresponding adult group. Data are presented as the mean \pm SEM. TNF, tumor necrosis factor; CA1, cornu ammonis 1; SP, stratum pyramidale; $\mathrm{SO}$, stratum oriens; $\mathrm{SR}$, stratum radiatum; tFI, transient forebrain ischemia; ROD, relative optical density.

immunoreactivity, we performed double immunofluorescence staining for TNF- $\alpha$ /GFAP (a marker for astrocyte), and we found that most of TNF- $\alpha$-immunoreactive non-pyramidal cells were colocalized with GFAP-immunoreactive astrocytes in the adult and young ischemia groups (Fig. 4).

Difference in tFI-induced change of TNF-R1 immunoreactivity between the adult and young. To examine the change of TNF-R1 immunoreactivity in CA1, immunohistochemistry was done in the adult and young groups (Fig. 5).

In the adult sham group, TNF-R1 immunoreactivity was easily shown in pyramidal neurons (Fig. 5A). In the adult ischemia group, TNF-R1 immunoreactivity was significantly 

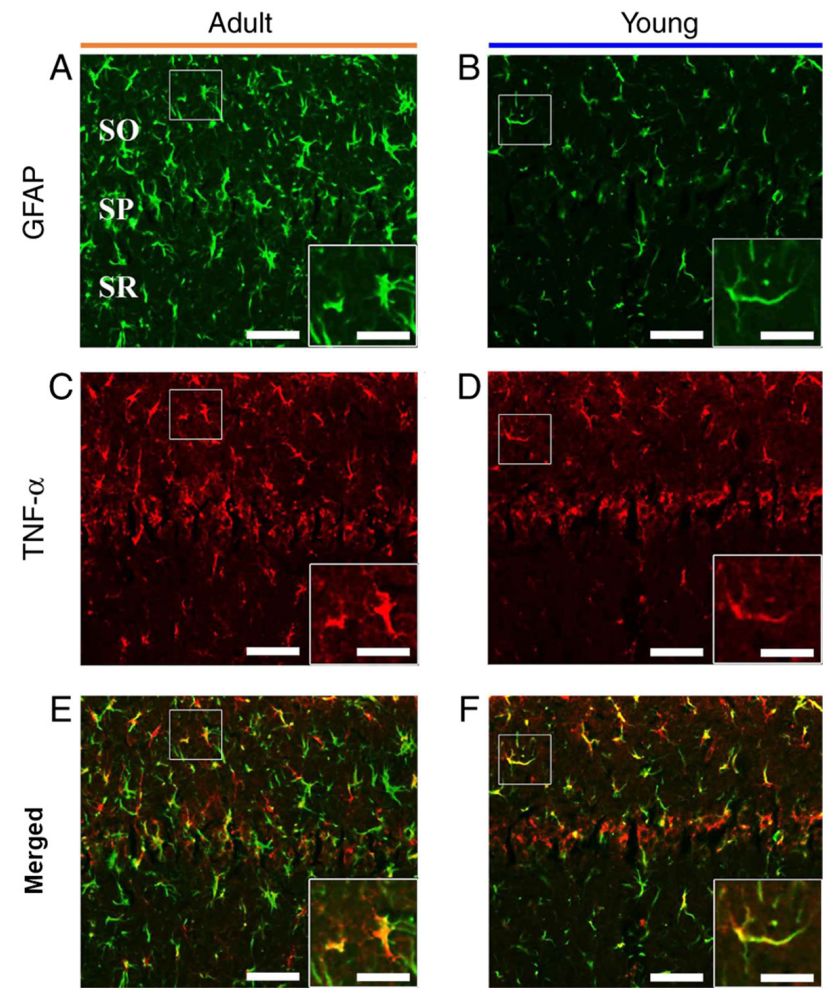

Figure 4. GFAP and TNF- $\alpha$ double immunofluorescence. Double immunofluorescence for (A and B) GFAP, (C and D) TNF- $\alpha$ and (E and F) merged images in the CA1 in the (A, C and $\mathrm{E})$ adult and $(\mathrm{B}, \mathrm{D}$ and $\mathrm{F})$ young ischemia groups 7 days after transient forebrain ischemia. GFAP immunoreactive astrocytes expressed TNF- $\alpha$ in both groups. Scale bar, $50 \mu \mathrm{m}$ (enlarged images, $100 \mu \mathrm{m}$ ). GFAP, glial fibrillary acidic protein; TNF, tumor necrosis factor; CA1, cornu ammonis 1; SO, stratum oriens; SP, stratum pyramidale; $\mathrm{SR}$, stratum radiatum.

increased (254.9\% of the adult sham group) in the pyramidal neurons at 1 day after tFI (Fig. 5C and K). Thereafter, TNF-R1 immunoreactivity in the pyramidal neurons was hardly shown at 4 days after tFI, but TNF-R1 immunoreactivity was newly shown in non-pyramidal cells, and TNF-R1 immunoreactivity in CA1 was $96.4 \%$ of the adult sham group (Fig. 5E and K). At 7 and 15 days after tFI, TNF-R1 immunoreactivity was more increased in the non-pyramidal cells, and TNF-R1 immunoreactivity in CA1 was 166.6 and $151.6 \%$ of the adult sham group, respectively (Fig. 5G, I and K). These non-pyramidal cells containing TNF-R1 immunoreactivity were identified as GFAP-immunoreactive astrocytes by double immunofluorescence (Fig. 6A, C and E).

In the young sham group, TNF-R1 immunoreactivity was also found in pyramidal neurons, and the immunoreactivity was similar to that in the adult sham group (Fig. 5B and K). In the young ischemia group, TNF-R1 immunoreactivity in the pyramidal neurons was increased only in the pyramidal neurons, showing that the TNF-R1 immunoreactivity was $93.0 \%$ at 1 day and $190.9 \%$ at 4 days after tFI compared with that in the corresponding adult ischemia group (Fig. 5D, F and K). At 7 and 15 days after $\mathrm{tFI}$, TNF-R1 immunoreactivity in CA1 was markedly decreased (68.1\% in the adult and $59.9 \%$ in the young) compared to that in the young sham group (Fig. 5H, J and K). At these times, TNF-R1 immunoreactivity was only shown in a few pyramidal neurons; TNF-R1 immunoreactivity was hardly detected in astrocytes by double immunofluorescence (Fig. 6B, D and F).

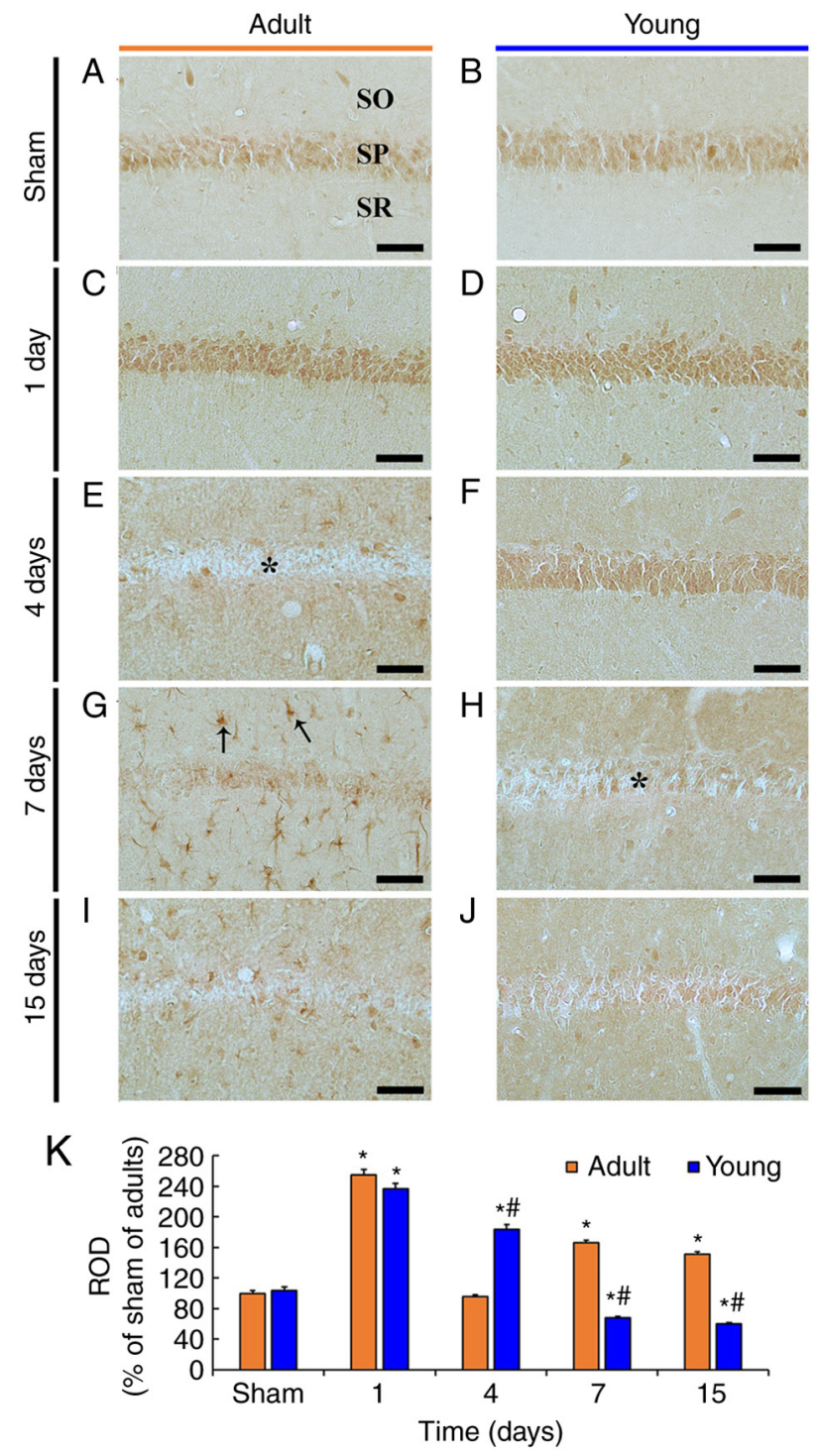

Figure 5. TNF-R1 immunohistochemistry. (A-J) TNF-R1 immunohistochemistry in the CA1 in the (A and B) sham and (C-J) ischemia groups of adult (left column) and young (right column) gerbils. In the adult ischemia group, TNF-R1 immunoreactivity increased in the SP (asterisk) 1 day after tFI and was rarely exhibited in the SP 4 days after tFI. TNF-R 1 immunoreactivity was present in non-pyramidal cells (arrows). In the young ischemia group, TNF-R1 immunoreactivity increased in the SP 4 days after tFI. Thereafter, TNF-R1 immunoreactivity weakened in the CA1. The asterisk in $(\mathrm{H})$ indicates that TNF-R1 immunoreactivity in the SP was exhibited in some pyramidal neurons. Scale bar, $50 \mu \mathrm{m}$. (K) ROD as \% of TNF-R1 immunoreactivity in CA1 $(\mathrm{n}=7)$ after tFI. ${ }^{*} \mathrm{P}<0.05$ vs. sham group; ${ }^{\#} \mathrm{P}<0.05$ vs. corresponding adult group. Data are presented as the mean \pm SEM. TNF-R1, tumor necrosis factor receptor 1; CA1, cornu ammonis 1; SP, stratum pyramidale; tFI, transient forebrain ischemia; ROD, relative optical density; $\mathrm{SO}$, stratum oriens; SR, stratum radiatum.

\section{Discussion}

In this study, we found that tFI-induced DND occurred in pyramidal neurons located in CA1 of the adult at 4 days after tFI, whereas the DND of pyramidal neurons in the young ischemia group was observed at 7 days after tFI. In addition, the number of dead pyramidal neurons (DND) at 7 and 15 days after tFI was significantly lower in the young ischemia group than that in the adult ischemia group. This result is consistent with our and other previous studies reporting that $\mathrm{tFI}$-induced 

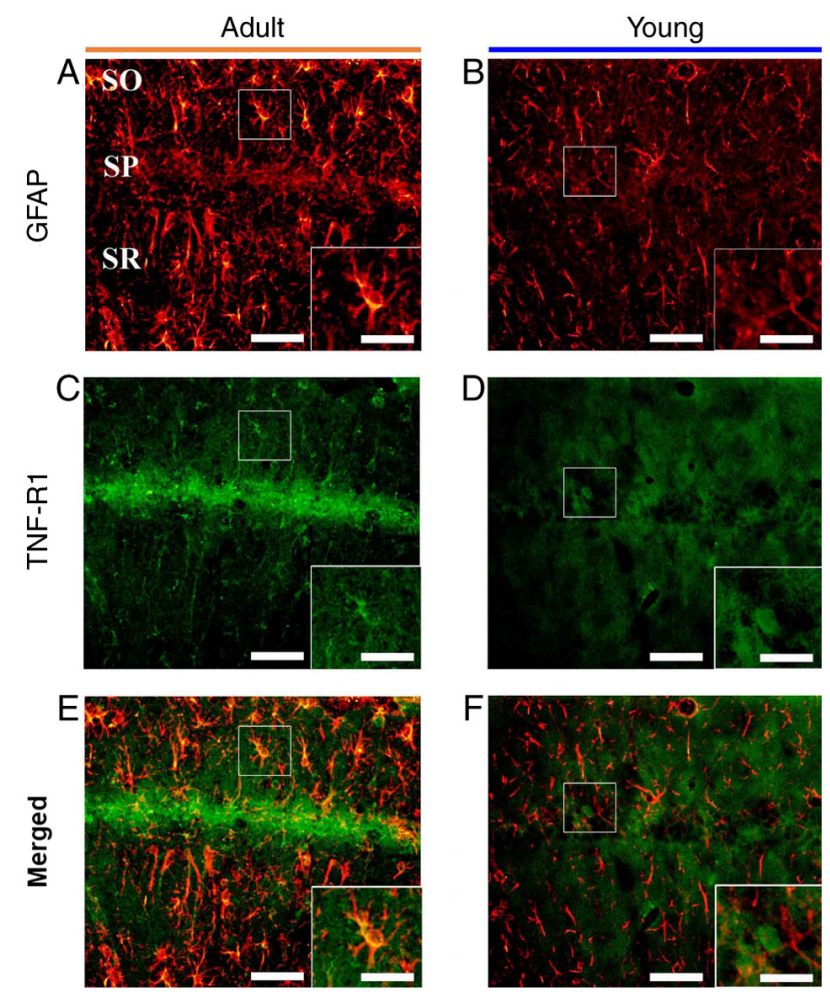

Figure 6. GFAP and TNF-R1 double immunofluorescence. Double immunofluorescence for (A and B) GFAP, (C and D) TNF-R1 and (E and F) merged images in the $\mathrm{CA} 1$ in the (A, C and $\mathrm{E})$ adult and $(\mathrm{B}, \mathrm{D}$ and $\mathrm{F})$ young ischemia groups 7 days after transient forebrain ischemia. GFAP immunoreactive astrocytes only expressed TNF-R1 in adults. Scale bar, $50 \mu \mathrm{m}$ (enlarged images, $100 \mu \mathrm{m}$ ). GFAP, glial fibrillary acidic protein; TNF-R1, tumor necrosis factor receptor 1; CA1, cornu ammonis 1; SO, stratum oriens; SP, stratum pyramidale; SR, stratum radiatum.

DND in the hippocampal CA1 in young gerbils was shown in fewer pyramidal neurons and more delayed in time than that shown in adult gerbils $(9,24,25)$. Taken together, we suggest that the difference of DND between young and adult groups might be associated with the differences of various factors to affect DND, including glial activation, inflammatory cytokine expression and NMDA receptor expression (26-28).

It has been widely accepted that both TNF- $\alpha$ and TNF-R1 expressions are highly increased in ischemic brains and upregulated TNF- $\alpha$ plays diverse roles in modulating cerebral ischemia-induced neuronal damage $(4,18,19,29-32)$. However, the exact role of TNF- $\alpha$ in ischemic brains is still controversial: Detrimental or protective role. A previous study showed that an intracerebroventricular injection of exogenous TNF- $\alpha$ led to neurological deficit and brain injury following focal brain ischemia induced by middle cerebral artery occlusion in rats, showing that the blockage of endogenous TNF- $\alpha$ by intracerebroventricular administration with a neutralized anti-TNF- $\alpha$ monoclonal antibody attenuated the focal ischemia-induced brain injury and infarct size compared with control rats (33). It was also reported that TNF- $\alpha$ was involved in oxygen-glucose deprivation-mediated cortical cell death in a dose-dependent manner (13). In contrast, Nawashiro et al (34) reported that intracisternal pretreatment with TNF- $\alpha$ reduced infarct size in a time- and dose-dependent manner in a mouse model of focal brain ischemia. In addition, it was reported that $\mathrm{TNF} \alpha$-expressing neurons were protected from glutamate-induced excitotoxicity in $\mathrm{TNF} \alpha$-transgenic mice compared with wild-type mice, and a pre-exposure of TNF- $\alpha$ to neurons resulted in protective effect against excitotoxicity in wild-type mice (35). Furthermore, it was reported that neuronal damage following focal cerebral ischemia or epileptic seizure was greater in TNFR-knockout mice than that in wild-type mice (36). Additionally, Lu et al (15) reported that kainic acid-induced seizure activity, and neuronal damage and glial activation in the hippocampus were significantly enhanced in TNF-R1-knockout mice compared with wild-type mice, suggesting that the protective role of TNF- $\alpha$ was mediated through TNF-R1 signaling. Therefore, in the present study, to disclose the reason why the tFI-induced DND of the CA1 pyramidal was different between adult and young gerbils, we investigated the changes of TNF- $\alpha$ and TNF-R1 protein expressions in the hippocampal CA1 region of adult and young gerbils after tFI. In our current study, TNF- $\alpha$ immunoreactivity was shown in the pyramidal neurons in both sham gerbils. After tFI, TNF- $\alpha$ immunoreactivity was gradually increased in the pyramidal neurons or astrocytes both groups, but the change pattern was different from each other and the immunoreactivity was higher in the young. TNF-R1 immunoreactivity in both sham groups was also shown only in the pyramidal neurons. After tFI, in both groups, TNF-R1 immunoreactivity was transiently enhanced in the pyramidal neurons at 1 day after tFI, thereafter, TNF-R1 immunoreactivity gradually decreased, showing that TNF-R1 immunoreactivity was newly expressed in astrocytes only in the adult group. Based on our and the above-mentioned previous studies, it can be postulated that a marked increase of TNF- $\alpha$ and TNF-R1 expression in the pyramidal neurons at early phase after tFI might be related to a protective potential against ischemic damage, and the different reduction of TNF- $\alpha$ and TNF-R1 expression might be associated with the difference in the tFI-induced DND of the pyramidal neurons between the adult and young.

It is well known that TNF- $\alpha$ is involved in inflammatory response and stimulates astrocyte and microglia activation at the site of ischemic injury following brain ischemic insults $(37,38)$. Reactive astrocytes have been known to be protective against ischemic injury by controlling immune response following cerebral ischemia (39) and to play an important role in the structural and funtional remodeling of ramainined brain tissue (40). In our current study, in the adult ischemia group, non-pyramidal cells located in SO and SR began to express TNF- $\alpha$ and TNF-R1 immunoreactivity from 4 days after tFI, and most of the TNF- $\alpha$ and TNF-R1 immunoreactive non-pyramidal cells were identified as astrocytes. Similar to this finding, TNF- $\alpha$ immunoreactivity was observed in astrocytes in human brain after ischemic stroke and traumatic brain injury $(41,42)$. In addition, it was reported that astrocytes expressing TNF- $\alpha$ and TNF-R1 were increased in the spinal cord after sciatic nerve injury in mice, suggesting that reactive astrocytes expressed more TNF- $\alpha$, which regulated the synthesis and expression of TNF- $\alpha$ through TNF-R1 on astrocytes in a positive feedback mechanism (43). Therefore, it is likely that an increased expression of TNF- $\alpha$ and TNF-R1 in activated astrocytes in the adult ischemia group might be related to the neurorestorative role of the reactive astrocytes after neuronal death induced by tFI. On the other hand, in the young ischemia group, TNF- $\alpha$-immunoreactive astrocytes began to be shown from 
7 days after tFI, and TNF-R1 immunoreactivity was found in the pyramidal neurons, not astrocytes, until 15 days after tFI. We cannot clearly explain why TNF-R1 immunoreactivity was not found in astrocytes at the late phase after tFI in the young ischemia group, which was apparently different from the adult ischemia group. For this finding, there is a paper showing that tFI-induced activation of astrocytes was much lower in young gerbils than that in adult gerbils between 4 and 15 days after tFI (28). Based on this finding, the decreased TNF-R1 expression in the pyramidal neurons in the young ischemia group at 7 days after tFI might be associated with more delayed DND; this DND was similar to that in the adult ischemia group at 4 days after tFI. Therefore, it can be mentioned that TNF-R1 expression in astrocytes following tFI might be dependent on difference in the degree of tFI-induced DND.

There are some limitations in the current study. We did not investigate how TNF- $\alpha$ and TNF-R1 expressions were affected by ischemia at various ages, such as 12 and 24 months. In addition, the levels of TNF- $\alpha$ and TNF-R1 expressions in serum and cerebrospinal fluid should be measured in future studies to support the conclusion of the present study. Furthermore, rodent researches should be extended to primate or human researches, which provide more convincing evidences of significant treatment strategy for patients with ischemic injuries.

In summary, both TNF- $\alpha$ and TNF-R1 protein levels and immunoreactivities were differently changed in ischemic hippocampal CA1 between young and adult gerbils following tFI. This result indicates that the different expressions of TNF- $\alpha$ and TNF-R1 may be one of underlying mechanisms of tFI-induced DND between the young and adult. Therefore, therapeutic strategy of ischemic insults must be considered in the age of patients with ischemic injury.

\section{Acknowledgements}

Not applicable.

\section{Funding}

The present study was supported by the Basic Science Research Program through the National Research Foundation of Korea (NRF) funded by the Ministry of Education (grant nos. NRF-2019R1A6A1A11036849 and NRF-2020R1F1A1052380).

\section{Availability of data and materials}

All data generated or analyzed during this study are included in this published article.

\section{Authors' contributions}

CHL and JHA drafted the initial manuscript. CHL, JHA, BHC, MHW and SYC were responsible for experimental conceptualization, data curation and analysis. BHC, DWK, HS, TKL and JHP performed the experiments, and collected and analyzed the data. MHW and SYC reviewed the manuscript for important intellectual content and acquired funding. MHW and SYC confirmed the authenticity of all the raw data. All authors have read and approved the final manuscript.

\section{Ethics approval and consent to participate}

All animal experiments were approved by the Institutional Animal Care and Use Committee (approval no. KW-200113-1).

\section{Patient consent for publication}

Not applicable.

\section{Competing interests}

The authors declare that they have no competing interests.

\section{References}

1. Kirino T: Delayed neuronal death in the gerbil hippocampus following ischemia. Brain Res 239: 57-69, 1982.

2. Farhadi Moghadam B and Fereidoni M: Neuroprotective effect of menaquinone-4 (MK-4) on transient global cerebral ischemia/ reperfusion injury in rat. PLoS One 15: e0229769, 2020.

3. Kim DW, Lee JC, Cho JH, Park JH, Ahn JH, Chen BH, Shin BN, Tae HJ, Seo JY, Cho JH, et al: Neuroprotection of ischemic preconditioning is mediated by anti-inflammatory, not pro-inflammatory, cytokines in the gerbil hippocampus induced by a subsequent lethal transient cerebral ischemia. Neurochem Res 40: 1984-1995, 2015.

4. Lee TK, Kang IJ, Kim B, Sim HJ, Kim DW, Ahn JH,Lee JC, Ryoo S, Shin MC, Cho JH, et al: Experimental pretreatment with chlorogenic acid prevents transient ischemia-induced cognitive decline and neuronal damage in the hippocampus through anti-oxidative and anti-inflammatory effects. Molecules 25: 3578, 2020.

5. Victoria ECG, Toscano ECB, Oliveira FMS, de Carvalho BA, Caliari MV, Teixeira AL, de Miranda AS and Rachid MA: Up-regulation of brain cytokines and metalloproteinases 1 and 2 contributes to neurological deficit and brain damage in transient ischemic stroke. Microvasc Res 129: 103973, 2020.

6. Lee CH, Yoo KY, Choi JH, Park OK, Hwang IK, Kim SK, Kang IJ, Kim YM and Won MH: Neuronal damage is much delayed and microgliosis is more severe in the aged hippocampus induced by transient cerebral ischemia compared to the adult hippocampus. J Neurol Sci 294: 1-6, 2010.

7. Lee TK, Kim H, Song M, Lee JC, Park JH, Ahn JH, Yang GE, Kim H, Ohk TG, Shin MC, et al: Time-course pattern of neuronal loss and gliosis in gerbil hippocampi following mild, severe, or lethal transient global cerebral ischemia. Neural Regen Res 14: 1394-1403, 2019.

8. Manwani B, Liu F, Scranton V, Hammond MD, Sansing LH and McCullough LD: Differential effects of aging and sex on stroke induced inflammation across the lifespan. Exp Neurol 249: 120-131, 2013.

9. Yan BC, Wang J, Cao J and Won MH: Less hippocampal neuronal death in young gerbils following transient global cerebral ischemia is associated with long-term maintenance of insulin-like growth factor 1 and its receptors in the hippocampal CA1 region. Mol Med Rep 17: 3055-3061, 2018.

10. Kinouchi K, Brown G, Pasternak G and Donner DB: Identification and characterization of receptors for tumor necrosis factor-alpha in the brain. Biochem Biophys Res Commun 181: 1532-1538, 1991.

11. Muñoz-Fernández MA and Fresno M: The role of tumour necrosis factor, interleukin 6 , interferon-gamma and inducible nitric oxide synthase in the development and pathology of the nervous system. Prog Neurobiol 56: 307-340, 1998.

12. Wajant H, Pfizenmaier K and Scheurich P: Tumor necrosis factor signaling. Cell Death Differe 10: 45-65, 2003.

13. Badiola N, Malagelada C, Llecha N, Hidalgo J, Comella JX, Sabriá J and Rodríguez-Alvarez J: Activation of caspase- 8 by tumour necrosis factor receptor 1 is necessary for caspase-3 activation and apoptosis in oxygen-glucose deprived cultured cortical cells. Neurobiol Dis 35: 438-447, 2009.

14. Doll DN, Rellick SL, Barr TL, Ren X and Simpkins JW: Rapid mitochondrial dysfunction mediates TNF-alpha-induced neurotoxicity. J Neurochem 132: 443-451, 2015.

15. Lu MO,Zhang XM, Mix E, Quezada HC, Jin T, Zhu J and Adem A: TNF-alpha receptor 1 deficiency enhances kainic acid-induced hippocampal injury in mice. J Neurosci Res 86: 1608-1614, 2008. 
16. Martin-Villalba A, Hahne M, Kleber S, Vogel J, Falk W, Schenkel J and Krammer PH: Therapeutic neutralization of CD95-ligand and TNF attenuates brain damage in stroke. Cell Death Differ 8: 679-686, 2001.

17. Wang X, Feuerstein GZ, Xu L, Wang H, Schumacher WA, Ogletree ML, Taub R, Duan JJ, Decicco CP and Liu RQ: Inhibition of tumor necrosis factor-alpha-converting enzyme by a selective antagonist protects brain from focal ischemic injury in rats. Mol Pharmacol 65: 890-896, 2004.

18. Lee JC, Park CW, Shin MC, Cho JH, Lee HA, Kim YM, Park JH, Ahn JH, Cho JH, Tae HJ, et al: Tumor necrosis factor receptor 2 is required for ischemic preconditioning-mediated neuroprotection in the hippocampus following a subsequent longer transient cerebral ischemia. Neurochem Int 118: 292-303, 2018.

19. Ohk TG, Ahn JH, Park YE, Lee TK, Kim B, Lee JC, Cho JH, Park JH, Won MH and Lee CH: Comparison of neuronal death and expression of TNF- $\alpha$ and MCT4 in the gerbil hippocampa CA1 region induced by ischemia/reperfusion under hyperthermia to those under normothermia. Mol Med Rep 22: 1044-1052, 2020

20. Ahn JH, Kim DW, Park JH, Lee TK, Lee HA, Won MH and Lee $\mathrm{CH}$ : Expression changes of CX3CL1 and CX3CR1 proteins in the hippocampal CA1 field of the gerbil following transient global cerebral ischemia. Int J Mol Med 44: 939-948, 2019.

21. Kanda I: Exotic Animal Formulary. Can Veterinary J 56: 736, 2015.

22. Park CW, Ahn JH, Lee TK, Park YE, Kim B, Lee JC, Kim DW, Shin MC, Park Y, Cho JH, et al: Post-treatment with oxcarbazepine confers potent neuroprotection against transient global cerebral ischemic injury by activating Nrf2 defense pathway. Biomed Pharmacother 124: 109850, 2020.

23. Schmued LC and Hopkins KJ: Fluoro-Jade B: A high affinity fluorescent marker for the localization of neuronal degeneration. Brain Res 874: 123-130, 2000.

24. Bae EJ, Chen BH, Yan BC, Shin BN, Cho JH, Kim IH, Ahn JH, Lee JC, Tae HJ, Hong S, et al: Delayed hippocampal neuronal death in young gerbil following transient global cerebral ischemia is related to higher and longer-term expression of p63 in the ischemic hippocampus. Neural Regen Res 10: 944-950, 2015.

25. Bertrand N, Ishii $\mathrm{H}$ and Spatz M: Cerebral ischemia in young and adult gerbils: Effects on cholinergic metabolism. Neurochem Int 28: 293-297, 1996

26. Seo JY, Yan BC, Park JH, Ahn JH, Kim IH, Lee JC, Kwon YG, Kim YM, Cho JH and Won MH: Comparison of the immunoreactivities of NMDA receptors between the young and adult hippocampal CA1 region induced by experimentally transient cerebral ischemia. J Neurol Sci 325: 108-114, 2013.

27. Yan BC, Kim SK, Park JH, Ahn JH, Lee CH, Yoo KY, Choi JH Lee DS, Kim MJ, Kim YM and Won MH: Comparison of inflammatory cytokines changes in the hippocampal CA1 region between the young and adult gerbil after transient cerebral ischemia. Brain Res 1461: 64-75, 2012.

28. Yan BC, Park JH, Ahn JH, Choi JH, Yoo KY, Lee CH, Cho JH, Kim SK, Lee YL, Shin HC and Won MH: Comparison of glial activation in the hippocampal CA1 region between the young and adult gerbils after transient cerebral ischemia. Cell Mol Neurobiol 32: 1127-1138, 2012.

29. Murakami Y, Saito K, Hara A, Zhu Y, Sudo K, Niwa M, Fujii H, Wada H, Ishiguro H, Mori $\mathrm{H}$ and Seishima M: Increases in tumor necrosis factor-alpha following transient global cerebral ischemia do not contribute to neuron death in mouse hippocampus. J Neurochem 93: 1616-1622, 2005.
30. Saito K, Suyama K, Nishida K, Sei Y and Basile AS: Early increases in TNF-alpha, IL-6 and IL-1 beta levels following transient cerebral ischemia in gerbil brain. Neurosci Lett 206: $149-152,1996$

31. Uno H, Matsuyama T, Akita $H$, Nishimura $H$ and Sugita $M$ : Induction of tumor necrosis factor-alpha in the mouse hippocampus following transient forebrain ischemia. J Cereb Blood Flow Metab 17: 491-499, 1997.

32. Xing $\mathbf{J}$ and Lu J: HIF-1 $\alpha$ activation attenuates IL- 6 and TNF- $\alpha$ pathways in hippocampus of rats following transient global ischemia. Cell Physiol Biochem 39: 511-520, 2016.

33. Barone FC, Arvin B, White RF, Miller A, Webb CL, Willette RN, Lysko PG and Feuerstein GZ: Tumor necrosis factor- $\alpha$ : A mediator of focal ischemic brain injury. Stroke 28: 1233-1244, 1997.

34. Nawashiro H, Tasaki K, Ruetzler CA and Hallenbeck JM: TNF-alpha pretreatment induces protective effects against focal cerebral ischemia in mice. J Cereb Blood Flow Metab 17: 483-490, 1997.

35. Marchetti L, Klein M, Schlett K, Pfizenmaier K and Eisel UL: Tumor necrosis factor (TNF)-mediated neuroprotection against glutamate-induced excitotoxicity is enhanced by N-methylD-aspartate receptor activation: Essential role of a TNF receptor 2-mediated phosphatidylinositol 3-kinase-dependent NF-kappa B pathway. J Biol Chem 279: 32869-32881, 2004.

36. Bruce AJ, Boling W, Kindy MS, Peschon J, Kraemer PJ, Carpenter MK, Holtsberg FW and Mattson MP: Altered neuronal and microglial responses to excitotoxic and ischemic brain injury in mice lacking TNF receptors. Nat Med 2: 788-794, 1996.

37. Watters $\mathrm{O}$ and $\mathrm{O}^{\prime}$ Connor JJ: A role for tumor necrosis factor- $\alpha$ in ischemia and ischemic preconditioning. J Neuroinflammation 8: 87, 2011.

38. Lai AY and Todd KG: Microglia in cerebral ischemia: Molecular actions and interactions. Can J Physiol Pharmacol 84: 49-59, 2006.

39. Wang R, Zhang X, Zhang J, Fan Y, Shen Y, Hu W and Chen Z: Oxygen-glucose deprivation induced glial scar-like change in astrocytes. PLoS One 7: e37574, 2012.

40. Liu Z and Chopp M: Astrocytes, therapeutic targets for neuroprotection and neurorestoration in ischemic stroke. Prog Neurobiol 144: 103-120, 2016.

41. Dziewulska D and Mossakowski M: Cellular expression of tumor necrosis factor a and its receptors in human ischemic stroke. Clin Neuropathol 22: 35-40, 2003.

42. Knoblach SM, Fan L and Faden AI: Early neuronal expression of tumor necrosis factor-alpha after experimental brain injury contributes to neurological impairment. J Neuroimmunol 95: 115-125, 1999.

43. Ohtori S, Takahashi K, Moriya H and Myers RR: TNF-alpha and TNF-alpha receptor type 1 upregulation in glia and neurons after peripheral nerve injury: Studies in murine DRG and spinal cord. Spine (Phila Pa 1976) 29: 1082-1088, 2004.

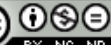

This work is licensed under a Creative Commons Attribution-NonCommercial-NoDerivatives 4.0 International (CC BY-NC-ND 4.0) License. 